\title{
Principais patógenos e susceptibilidade in vitro antimicrobiana em ceratites bacterianas: Revisão de cinco anos, 2005 a 2009
}

\author{
Main pathogens and in vitro antimicrobial susceptibility in bacterial keratitis: \\ 5-year study, 2005-2009
}

Guilherme Andrade do Nascimento Rocha ${ }^{1}$, Reinaldo Ferreira da Silva ${ }^{1}$, Mayana Freitas Lopes ${ }^{1}$, Nicolas Cesário Pereira ${ }^{1}$, Luciene Barbosa de Sousa ${ }^{1}$

\section{RESUMO}

Objetivo: Determinar os principais patógenos envolvidos na etiologia das ceratites bacterianas no Hospital Oftalmológico de Sorocaba/SP e a susceptibilidade in vitro dos principais antimicrobianos utilizados no tratamento desta afeç̧ão ocular.

Métodos: Estudo retrospectivo de todos os prontuários submetidos a exame microbiológico por suspeita de ceratite infecciosa, atendidos no Hospital Oftalmológico de Sorocaba/SP, entre 2005 e 2009. Para testar a susceptibilidade dos antimicrobianos, foram realizados antibiogramas através do método padrão de difusão em discos e a interpretação da sensibilidade foi realizada seguindo-se a padronização do National Committee on Clinical Laboratory Standards (NCCLS, Villanova, PA, USA). Resultados: De 963 coletas, 278 (28,86\%) foram positivas para algum patógeno. Dentre as coletas positivas para bactérias, os principais patógenos identificados foram: Staphylococcus aureus (30.56\%), Staphylococcus epidermidis(30,56\%), Streptococcus sp (9,43\%) e Pseudomonas sp (9,43\%). No total, 191 (72,08\%) dos patógenos identificados eram Gram-positivos e 74 (27,92\%) eram Gram-negativos. O exame laboratorial evidenciou altos índices de sensibilidade para a maioria dos antimicrobianos (acima de $85 \%)$, com exceção da cefalotina $(53,13 \%)$. Os maiores índices de resistência foram observados com a cefalotina $(33,47 \%)$ e a ciprofloxacina $(11,36 \%)$. Conclusões: Bactérias Gram-positivas foram as principais isoladas nos exames microbiológicos neste estudo, com número expressivo de estafilococos, apesar de um crescente número de bactérias Gram-negativas como principais patógenos isolados, principalmente representado por Pseudomonas, relacionado ao uso disseminado de lentes de contato. O exame laboratorial evidenciou altos índices de sensibilidade para a maioria dos antimicrobianos (acima de $85 \%$ ), com exceção da cefalotina $(53,13 \%)$. Os maiores índices de resistência foram observados com a cefalotina $(33,47 \%)$ e a ciprofloxacina $(11,36 \%)$. Diante desta variabilidade importante tanto de principais patógenos envolvidos na etiologia da ceratite bacteriana como de susceptibilidade antimicrobiana, torna-se muito importante saber qual o perfil das infecções com as quais estamos lidando, para que tal afecção seja conduzida da maneira correta, seguindo-se a individualidade de cada localidade.

Descritores: Infecções oculares bacterianas/microbiologia; Testes de sensibilidade microbiana; Ceratite/microbiologia; Resistência microbiana a drogas

\begin{abstract}
Purpose: To determine the most common pathogens involved in the etiology of bacterial keratitis at the Sorocaba Ophthalmological Hospital and the in vitro susceptibility of main antimicrobial used in the treatment of this pathology.

Methods: Retrospective study of all patients suspected of infectious keratitis that underwent microbiological exam at the Sorocaba Ophthalmological Hospital, between 2005 and 2009. To test susceptibility of antimicrobial drugs, antibiograms were made through the standard method of discs diffusion and interpretation following the National Committee on Clinical Laboratory Standards (NCCLS, Villanova, PA, USA).

Results: 963 samples were obtained in this period and 278 (28.86\%) were positive for some pathogen. Among the positive for bacteria, the most common were Staphylococcus aureus (30.56\%), Staphylococcus epidermidis (30.56\%), Streptococcus sp (9.43\%) and Pseudomonas sp (9.43\%). Dividing in Gram's method, 191 (72.08\%) were Gram-positive and 74 (27.92\%) were Gram-negative. Analyzing in vitro antimicrobial effectiveness, the majority presented good susceptibility rates, beyond $85 \%$, except cephalotin which presented $53.13 \%$. At the same analysis, higher resistance rates with cephalotin (33.47\%) and ciprofloxacin (11.36\%) were noted.

Conclusion: Gram-positive bacteria were the most common pathogen isolated at microbiologic exams, especially staphylococci. despite the increasing number of isolated Gram-negative bacteria, meanly represented by Pseudomonas, related with the widespread use of contact lenses. Laboratorial exam revealed high sensibility values for most of antibiotics (more than 85\%), except for cephalotin (53.13\%). Higher resistance values were observed with cephalotin (33.47\%) and ciprofloxacin (11.36\%). With all these important variability of antibiotic susceptibility and pathogens involved in bacterial keratitis etiology, it is very important to know the specific characteristics of each infection for a better patient casre.
\end{abstract}

Keywords: Eye infections, bacterial/microbiology; Microbial sensitivity tests; Keratitis/microbiology; Drug resistance, microbial
Trabalho realizado no Setor de Doenças Externas e Córnea e Cirurgia Refrativa no Hospital Oftalmológico de Sorocaba - Sorocaba (SP), Brasil.

Médicos, Setor de Doenças Externas e Córnea e Cirurgia Refrativa, Hospital Oftalmológico de Sorocaba - Sorocaba (SP), Brasil.

Endereço para correspondência: Guilherme A. N. Rocha. Rua Nabeck Shiroma, 210 - Jardim Emília - Sorocaba (SP) - CEP 18031-060 - E-mail: drguilhermerocha@hotmail.com

Recebido para publicação em 01.06.2010

Última versão recebida em 06.10.2010

Aprovação em 25.11.2010

Nota Editorial: Depois de concluída a análise do artigo sob sigilo editorial e com a anuência do Dr. Roberto Freda sobre a divulgação de seu nome como revisor, agradecemos sua participação neste processo.

\section{INTRODUÇÃO}

C eratite bacteriana é uma condição comum e prevenível de morbidade visual. Alguns casos apresentam aparecimento rápido e inflamação estromal progressiva. A gravidade da infecção depende da condição corneana prévia e da patogenicidade do micro-organismo envolvido. Se não tratada, normalmente leva à destruição tecidual progressiva com perfuração corneana ou extensão para tecidos adjacentes.

Uma grande variedade de patógenos pode estar envolvida em ceratites. A prevalência de um organismo depende da localização geográfica, fatores climáticos e de fatores de risco para a infecção(1-4). Até recentemente, a maioria dos casos estava relacionada a traumas oculares e/ou doenças ou cirurgias da superfície ocular. Entretanto, o uso indiscriminado de lentes de contato aumentou consideravelmente a incidência de ceratite nestas pessoas, passando a ser o 


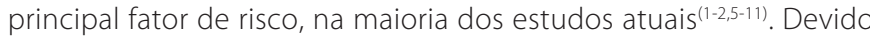
esta ampla variedade de agentes causadores, muitos com espectro de sensibilidade a antimicrobianos restritos, o oftalmologista deve considerar o uso de testes diagnósticos microbiológicos em casos selecionados ${ }^{(12-14)}$.

Atualmente, não há um agente antimicrobiano efetivo contra todos os patógenos causadores de ceratites. O uso de terapia empírica de amplo espectro é recomendado até que o micro-organismo envolvido seja identificado ${ }^{(1,3-5,7,15)}$. Apesar disto, a modificação da terapia inicial deve basear-se na resposta clínica e não no resultado de sensibilidade antimicrobiana. A partir da ausência de melhora clínica, a utilização de um regime alternativo deve ser considerada, orientada pelo resultado do exame $e^{(1,3,7,15)}$

Este trabalho tem como objetivo determinar os principais patógenos envolvidos na etiologia das ceratites bacterianas e a susceptibilidade in vitro dos principais antimicrobianos utilizados no tratamento desta afecção ocular.

\section{MÉTODOS}

Estudo retrospectivo de todos os prontuários submetidos a exame microbiológico por quadro suspeito de ceratite infecciosa, atendidos no Hospital Oftalmológico de Sorocaba/SP, no período de 2005 e 2009. Os exames microbiológicos foram realizados conforme os seguintes critérios: (1) lesões $\geq 3$ mm; (2) próxima ao eixo visual; (3) perfuração ou iminência de perfuração; (4) comprometimento escleral; (5) utilização prévia de antimicrobianos sem resposta clínica; (6) história prévia de doenças oculares.

Os raspados foram feitos com auxílio de espátula de Kimura flambada na chama de lamparina e esfriada em solução estéril, à lâmpada de fenda com auxílio de blefarostato e após instilação de cloridrato de proximetacaína 0,5\%. O material colhido foi destinado para: (1) cultura, semeado nas placas de ágar-sangue e ágar-chocolate; (2) bacterioscopia citológica, coradas por Gram e Giemsa; (3) meio de tioglicolato, para anaeróbios e (4) cultura para fungos em placa de ágar-Sabouraud. Durante a coleta, o material necrótico era desprezado e as amostras colhidas das bordas e da região profunda da lesão.

Para testar a susceptibilidade dos antimicrobianos, foram realizados antibiogramas através do método padrão de difusão em discos e a interpretação da sensibilidade foi realizada seguindo-se a padronização do National Committee on Clinical Laboratory Standards (NCCLS, Villanova, PA, USA).

Como o exame é realizado em laboratório terceirizado, diversos antibióticos foram testados. Assim, para análise estatística, somente antibióticos com aplicação em oftalmologia foram considerados.

\section{RESULTADOS}

Foram realizadas 963 coletas no período entre 2005 e 2009, seguindo o critério estabelecido. Destas, 278 coletas (28,86\%) foram positivas para algum patógeno, sendo 265 (95,33\%) bacterianas e 13 (4,67\%) fúngicas. Os resultados estão resumidos na tabela 1.

Dentre as coletas positivas para bactérias, os principais patógenos identificados foram: Staphylococcus aureus (30,56\%), Staphylococcus epidermidis (30,56\%), Streptococcus sp $(9,43 \%)$ e Pseudomonas sp (9,43\%). No total, 191 (72,08\%) dos patógenos identificados eram Gram-positivos e 74 (27,92\%) eram Gram-negativos.

Todas as bactérias encontradas estão descritas na tabela 2.

Através da análise dos antibiogramas, foi estudada a susceptibilidade bacteriana. Separamos conforme o método de Gram e, individualmente, avaliamos as quatro bactérias mais prevalentes nas amostras.

Os resultados estão descritos nas tabelas 3 e 4.

\section{DISCUSSÃO}

O fato de a ceratite infecciosa ser um quadro oftalmológico comum faz com que seu correto diagnóstico e tratamento sejam de extrema importância, diante das complicações e sequelas passíveis de ocorrer.

A identificação do patógeno através de exames microbiológicos constitui um passo importante, mas não indispensável. Trabalhos que estudaram a importância da realização de culturas demonstraram que a grande maioria das úlceras com patógenos adquiridos na comunidade, respondem bem à monoterapia com antibióticos de amplo espectro. Assim, sugerem que as culturas devem ser realizadas apenas em casos selecionados ${ }^{(12-14)}$. Em nosso trabalho, alguns critérios foram obedecidos para realização dos exames, como mostrado anteriormente.

Entretanto, nem sempre é possível identificar o agente causador, e isto não deve retardar o tratamento. Em nossa estatística, obtivemos $28.86 \%$ de positividade, valor abaixo dos valores médios encontrados na literatura $(1,3-6,8,10-11,15-17)$, havendo resultados de até $85 \%-86 \%$ de positividade ${ }^{(1,16)}$. Diante disto é importante questionarmos nossos índices, no intuito de melhorar a positividade, para uma melhor abordagem diagnóstica e terapêutica. Devemos lembrar que alguns fatores são capazes de alterar o resultado dos exames, como utilização prévia de antibióticos ${ }^{(1,5,7)}$; uso inadvertido pelo paciente ou uso durante a coleta de colírio anestésico com preservativos, que tem ação bactericida, diminuindo o número de patógenos ${ }^{(5,18)}$; amostra de material pequena; transporte e armazenagem inadequados; cultivo em meios inadequados.

As principais bactérias isoladas foram Staphylococcus aureus, Staphylococcus epidermidis, Streptococcus sp e Pseudomonas sp, sendo os dois primeiros os mais encontrados (30,56\% cada). Os patógenos Gram-positivos somaram 73,23\% e os Gram-negativos, 26,77\%, com Pseudomonas sp sendo o principal Gram-negativo isolado, com 9,43\%. Na maioria das séries, o principal patógeno isolado também foi Staphylococcus $\mathrm{sp}^{(1,3-5,10,16,19-20)}$, mas em outros houve predomínio de Pseudomonas $\mathrm{sp}^{(6,9,11,17,21-22)}$. Este aumento tem provável relação com a maior participação do uso de lentes de contato como fator de risco, pois Pseudomonas sp é o principal micro-organismo isolado nestes $\operatorname{casos}^{(1,5-9,11,22)}$. Quando analisamos

Tabela 1. Distribuição das coletas microbiológicas por período

\begin{tabular}{ccccc}
\hline Ano & Coletas & Positivas (\%) & Bacterianas & Fúngicas \\
\hline 2005 & 87 & $44(50,57)$ & 44 & - \\
2006 & 143 & $50(34,96)$ & 48 & 2 \\
2007 & 186 & $47(25,26)$ & 47 & - \\
2008 & 210 & $70(33,33)$ & 59 & 11 \\
2009 & 337 & $67(19,88)$ & 67 & - \\
Total & 963 & $278(28,86)$ & 265 & 13 \\
\hline
\end{tabular}

Tabela 2. Principais patógenos isolados

\begin{tabular}{lrr}
\hline Bactéria isolada & N & \% \\
\hline Staphylococcus aureus & 81 & 30,56 \\
Staphylococcus epidermidis & 81 & 30,56 \\
Streptococcus sp & 25 & 9,43 \\
Pseudomonas aeruginosa & 25 & 9,43 \\
Klebsiella sp & 15 & 5,66 \\
Escherichia coli & 13 & 4,90 \\
Proteus sp & 7 & 2,64 \\
Enterobacter sp & 5 & 1,88 \\
Bacillus sp & 3 & 1,13 \\
Enterococcus sp & 4 & 1,50 \\
Acinetobacter sp & 3 & 1,13 \\
Serratia sp & 1 & 0,37 \\
Micrococcussp & 1 & 0,37 \\
Citrobacter sp & 1 & 0,37 \\
Total & 265 & 100,00 \\
\hline
\end{tabular}


estudos brasileiros, os resultados são similares aos nossos, com predomínio de Staphylococcus sp ${ }^{(10,19-20)}$, o que mostra uma real influência geográfica na etiologia das ceratites.

Os testes de susceptibilidade in vitro devem ser analisados cuidadosamente ${ }^{(12-14)}$. É importante lembrar que o comportamento de uma bactéria in vitro não corresponde necessariamente a seu comportamento in vivo, daí a importância da resposta clínica no seguimento terapêutico.

Analisando a atuação dos antimicrobianos, a maioria apresenta bons índices de sensibilidade, acima de 85\%, exceto pela cefalotina, que apresenta índice de sensibilidade de 53,13\%. Na mesma análise, observam-se maiores índices de resistência para cefalotina $(33,47 \%)$ e ciprofloxacina (11,36\%).

Dividindo os patógenos conforme o método de Gram, o resultado não foi diferente:

- Gram-positivos: a maioria dos antimicrobianos mantém bons índices de sensibilidade, superiores a 88\%, com exceção da cefalotina, que apresenta 66,08\%. Avaliando-se os índices de resistência, a cefalotina permanece com os maiores índices, $19,88 \%$, seguida pela ciprofloxacina, com $8,64 \%$.
- Gram-negativos: a maioria continua com bons índices de sensibilidade, acima de $80 \%$, exceto pela cefalotina e cloranfenicol, com $20,28 \%$ e $56,36 \%$, respectivamente. Entretanto, os índices de resistência são maiores para todos os antibióticos estudados, sendo a cefalotina, cloranfenicol e ciprofloxacina, respectivamente com 68,11\%, 20\% e 18,03\%, os maiores índices.

No estudo individual de cada bactéria, alguns pontos merecem atenção:

- Staphylococcus aureus apresentou boa sensibilidade a todos antibióticos estudados, com resistência pouco maior à ciprofloxacina (10,14\%) e tobramicina (10,12\%);

- Staphylococcus epidermidis apresentou maior sensibilidade que Staphylococcus aureus, porém apresentou baixa sensibilidade $(49,31 \%)$ e maior resistência $(34,24 \%)$ à cefalotina, teoricamente um bom agente contra Gram-positivos;

- Streptococcus sp também demonstrou boa sensibilidade à maioria dos agentes testados, exceto, novamente, pela cefalotina, com 52,17\% de sensibilidade e 34,78\% de resistência;

- Pseudomonas sp apresentou baixos índices de sensibilidade e altos índices de resistência perante a maioria dos anti-

Tabela 3. Susceptibilidade dos patógenos aos antimicrobianos

\begin{tabular}{|c|c|c|c|c|c|c|c|c|c|c|c|c|c|c|c|}
\hline \multirow[b]{2}{*}{ Antibiótico } & \multicolumn{5}{|c|}{ Gram-positivos } & \multicolumn{5}{|c|}{ Gram-negativos } & \multicolumn{5}{|c|}{ Total } \\
\hline & $\mathbf{N}$ & $\mathbf{S}$ & $\mathbf{R}$ & $\% \mathrm{~S}$ & $\% \mathbf{R}$ & $\mathbf{N}$ & $S$ & $\mathbf{R}$ & $\% \mathrm{~S}$ & $\% \mathbf{R}$ & $\mathbf{N}$ & $\mathbf{S}$ & $\mathbf{R}$ & $\% \mathrm{~S}$ & $\%$ R \\
\hline Cefalotina & 171 & 113 & 34 & 66,08 & 19,88 & 69 & 14 & 47 & 20,28 & 68,11 & 239 & 127 & 80 & 53,13 & 33,47 \\
\hline Gentamicina & 189 & 177 & 6 & 93,65 & 3,17 & 67 & 58 & 8 & 86,56 & 11,94 & 256 & 235 & 14 & 91,79 & 5,46 \\
\hline Amicacina & 190 & 169 & 8 & 88,94 & 4,21 & 68 & 57 & 5 & 83,82 & 7,35 & 258 & 226 & 13 & 87,59 & 5,03 \\
\hline Vancomicina & 148 & 148 & - & 100,00 & - & 3 & 3 & - & 100,00 & - & 151 & 151 & - & 100,00 & - \\
\hline Neomicina & 165 & 146 & 5 & 88,48 & 3,03 & 54 & 47 & 3 & 87,03 & 5,55 & 219 & 193 & 8 & 88,12 & 3,65 \\
\hline Ciprofloxacina & 162 & 146 & 14 & 90,12 & 8,64 & 61 & 50 & 11 & 81,96 & 18,03 & 220 & 193 & 25 & 87,72 & 11,36 \\
\hline Cloranfenicol & 147 & 142 & 2 & 96,59 & 1,36 & 55 & 31 & 11 & 56,36 & 20,00 & 202 & 173 & 13 & 85,64 & 6,43 \\
\hline Tobramicina & 180 & 166 & 12 & 92,22 & 6,66 & 67 & 55 & 9 & 82,08 & 18,00 & 248 & 221 & 21 & 89,11 & 8,46 \\
\hline Gatifloxacina & 59 & 59 & - & 100,00 & - & 17 & 15 & 2 & 83,33 & 13,43 & 76 & 74 & 2 & 97,36 & 2,63 \\
\hline
\end{tabular}

$\mathrm{N}=$ número de culturas em que $\mathrm{o}$ antibiótico foi testado; $\mathrm{S}=$ sensível; $\mathrm{R}=$ resistente;\% $\mathrm{S}=$ porcentual de sensibilidade= $\mathrm{S} / \mathrm{N} ; \% \mathrm{R}=\mathrm{porcentual}$ de resistência= $\mathrm{R} / \mathrm{N}$

Tabela 4. Susceptibilidade antimicrobiana detalhada dos principais patógenos isolados

\begin{tabular}{|c|c|c|c|c|c|c|c|c|c|c|}
\hline & \multicolumn{5}{|c|}{ Staphylococcus aureus (81) } & \multicolumn{5}{|c|}{ Staphylococcus epidermidis (81) } \\
\hline & $\mathbf{N}$ & $\mathbf{S}$ & $\mathbf{R}$ & $\% \mathbf{S}$ & $\% \mathbf{R}$ & $\mathbf{N}$ & $\mathbf{S}$ & $\mathbf{R}$ & $\% \mathbf{S}$ & $\% \mathbf{R}$ \\
\hline Cefalotina & 67 & 59 & 1 & 88,05 & 1,49 & 73 & 36 & 25 & 49,31 & 34,24 \\
\hline Gentamicina & 80 & 75 & 3 & 93,75 & 3,75 & 79 & 75 & 3 & 94,93 & 3,79 \\
\hline Amicacina & 75 & 60 & 5 & 80,00 & 6,67 & 79 & 76 & 1 & 96,20 & 1,26 \\
\hline Vancomicina & 70 & 70 & - & 100,00 & - & 71 & 71 & - & 100,00 & - \\
\hline Neomicina & 63 & 52 & 2 & 82,53 & 3,17 & 71 & 69 & - & 97,18 & - \\
\hline Ciprofloxacina & 69 & 62 & 7 & 89,85 & 10,14 & 71 & 64 & 7 & 90,14 & 9,85 \\
\hline Cloranfenicol & 59 & 56 & 1 & 94,91 & 1,69 & 58 & 57 & - & 98,27 & - \\
\hline Tobramicina & 79 & 70 & 8 & 88,60 & 10,12 & 71 & 69 & - & 97,18 & - \\
\hline \multirow[t]{3}{*}{ Gatifloxacina } & 24 & 24 & - & 100,00 & - & 21 & 21 & - & 100,00 & - \\
\hline & \multicolumn{5}{|c|}{ Streptococcus sp (25) } & \multicolumn{5}{|c|}{ Pseudomonas sp (25) } \\
\hline & $\mathbf{N}$ & $\mathbf{S}$ & $\mathbf{R}$ & $\% \mathbf{S}$ & $\% \mathbf{R}$ & $\overline{\mathbf{N}}$ & $\mathbf{S}$ & $\mathbf{R}$ & $\% \mathbf{S}$ & $\overline{\%} \mathbf{R}$ \\
\hline Cefalotina & 23 & 12 & 8 & 52,17 & 34,78 & 25 & 2 & 22 & 8,00 & 88,00 \\
\hline Gentamicina & 22 & 20 & 1 & 90,90 & 4,54 & 23 & 17 & 5 & 73,91 & 21,73 \\
\hline Amicacina & 24 & 21 & 2 & 87,50 & 8,33 & 25 & 21 & 4 & 84,00 & 16,00 \\
\hline Vancomicina & 7 & 7 & - & 100,00 & - & - & - & - & - & - \\
\hline Neomicina & 23 & 17 & 3 & 73,91 & 13,04 & 22 & 15 & 3 & 68,19 & 13,64 \\
\hline Ciprofloxacina & 17 & 15 & - & 88,23 & - & 20 & 14 & 6 & 70,00 & 30,00 \\
\hline Cloranfenicol & 23 & 22 & 1 & 95,65 & 4,34 & 20 & 3 & 10 & 15,00 & 50,00 \\
\hline Tobramicina & 23 & 20 & 2 & 86,95 & 8,69 & 25 & 19 & 6 & 76,00 & 24,00 \\
\hline Gatifloxacina & 9 & 9 & - & 100,00 & - & 7 & 5 & 2 & 71,42 & 28,57 \\
\hline
\end{tabular}

$($ )= quantidade de culturas positivas para cada patógeno; $\mathrm{N}=$ número de culturas em que o antibiótico foi testado; $\mathrm{S}=$ sensível; $\mathrm{R}=$ resistente; $\% \mathrm{~S}=$ porcentual de sensibilidade= $\mathrm{S} / \mathrm{N} ; \% \mathrm{R}=$ porcentual de resistência= $\mathrm{R} / \mathrm{N}$ 
bióticos testados. Apenas $8 \%$ das cepas isoladas foram sensíveis à cefalotina, enquanto $88 \%$ foram resistentes. Amicacina foi o antimicrobiano com melhor perfil de sensibilidade (84\%), apesar de 16\% de resistência.

Quando comparamos nossos resultados com outros estudos, percebemos a ampla variabilidade de patógenos e susceptibilidade aos antimicrobianos:

- Schaefer et al. ${ }^{(1)}$ encontrou resultados semelhantes de amostras resistentes à cefalosporinas e fluorquinolonas, com 37\% e 1-15\%, respectivamente. Já as amostras resistentes ao cloranfenicol e aminoglicosídeos apresentaram resultados maiores, com 18\% e 13-22\%, respectivamente.

- Butler et al. ${ }^{(4)}$ obteve taxa de sensibilidade à cefalotina bem diferente da nossa, não sendo de 100\% apenas para Staphylococcus coagulase-negativo, com 78,3\%. Outra diferença foi a ausência de resistência à ciprofloxacina e gentamicina por Pseudomonas. Já para ciprofloxacina, houve algumas amostras de Staphylococcus resistentes, mas a maioria foi sensível, com resultados bem próximos aos nossos.

- Yeh et al. ${ }^{(15)}$ obteve $24 \%$ de resistência à cefalosporina nas amostras testadas de $S$. aureus e $<5 \%$ nos antibióticos testados para Gram-negativos. Estes resultados foram opostos aos nossos, pois obtivemos apenas 1.49\% de resistência à cefalotina nas amostras de $S$. aureus e todos antibióticos testados obtiveram >5\% de resistência aos Gram-negativos.

- Tan et al..$^{(9)}$ encontrou todos Gram-positivos sensíveis às cefalosporinas e apenas um Gram-negativo resistente à gentamicina. Obteve também todos os casos de Pseudomonas e 33,33\% dos Gram-positivos resistentes ao cloranfenicol. Em nossa série, obtivemos também bons resultados com gentamicina para os Gram-negativos, mas não com as cefalosporinas para os Gram-positivos. No caso do cloranfenicol, a resistência das amostras de Pseudomonas foi a segunda maior (50\%), mas a resistência dos Gram-positivos foi muito pequena $(1,36 \%)$

Cada estudo utilizou um tipo de tratamento, mas a maioria concorda com a necessidade de um tratamento inicial de amplo espectro, com uma medicação específica para patógenos Gram-positivos e outra para Gram-negativos. O esquema considerado "padrãoouro" no tratamento das ceratites bacterianas é a combinação de gentamicina e uma cefalosporina ${ }^{(23)}$. Entretanto, é demonstrado que a concentração de gentamicina diminui rapidamente e que apresenta baixa penetração ocular ${ }^{(1)}$. Associado a isso, há a toxicidade epitelial com uso dos antibióticos fortificados. Desta forma, as fluorquinolonas começaram a ser estudadas, no intuito de comprovar a eficácia e segurança de uma monoterapia.

O uso das fluorquinolonas no tratamento das ceratites bacterianas aumentou bastante nos últimos anos, principalmente após alguns estudos demonstrarem que o uso de ciprofloxacina ou ofloxacina 0.3\% em monoterapia apresentou resultados semelhantes ou meIhores em eficácia quando comparados à associação cefalosporina e aminoglicosídeo ${ }^{(21,24-25)}$. Entretanto, o principal argumento que impede o uso de monoterapia de fluorquinolona como primeira $1^{\underline{a}}$ escolha no tratamento de ceratites bacterianas é o registro por vários estudos do aparecimento de amostras resistentes, principalmente contra Streptococcus sp ${ }^{(1,26-27)}$, S. aureus ${ }^{(27)}$, Staphylococcus coagulasenegativo ${ }^{(1,26,28)}$ e Pseudomonas sp $^{(29)}$. Uma provável alternativa para monoterapia seria a utilização de quinolonas de $3^{\underline{a}}$ e $4^{\underline{a}}$ geração, que apresentam perfil maior de cobertura para bactérias Gram-positivas. Em nosso estudo, a gatifloxacina apresentou 97,36\% de sensibilidade às amostras, apenas com duas amostras resistentes, ambas de Pseudomonas sp.

Em nosso serviço, utilizamos como protocolo para todo caso onde é realizada coleta de exame microbiológico, a associação de cefalotina e ofloxacina como primeira linha de tratamento. Esta es- colha é baseada no fato das cefalosporinas terem um bom perfil de atuação contra bactérias Gram-positivas, e fazer parte do "padrãoouro" de tratamento ${ }^{(23)}$ e as fluorquinolonas apresentarem eficácia comprovada, similar à associação de fortificados ${ }^{(21,24-25)}$, apesar de apresentar resistência principalmente com bactérias Gram-positivas, fato teoricamente coberto pelas cefalosporinas. Tal esquema só é alterado diante de ausência de melhora clínica, utilizando-se o resultado do antibiograma para escolha do novo esquema terapêutico.

\section{CONCLUSÃO}

Ceratite bacteriana continua sendo uma condição com tratamento desafiador e de ameaça visual importante. O rápido isolamento e início de terapia antimicrobiana constituem passos importantes no manejo de tal patologia.

Bactérias Gram-positivas foram as principais isoladas em exames microbiológicos neste estudo, com número expressivo de estafilococos, apesar de um crescente número de bactérias Gram-negativas como principais patógenos isolados, principalmente representado por Pseudomonas, relacionado ao uso disseminado de lentes de contato.

Quando realizamos um estudo como este, em que realizamos uma comparação com outros estudos de diversas partes do mundo e que fica evidente uma variabilidade importante tanto de principais patógenos envolvidos na etiologia da ceratite bacteriana como de susceptibilidade antimicrobiana, torna-se muito importante saber qual o perfil das infecções com as quais estamos lidando, para que tal patologia seja conduzida da maneira correta, seguindo-se a individualidade de cada localidade.

Avaliando-se nossos resultados in vitro, chama a atenção de que os dois antibióticos utilizados como primeira escolha no tratamento em nosso serviço, cefalosporina + fluorquinolona, apresentam as maiores taxas de resistência, tanto quando se leva em consideração os patógenos em geral, ou quando são divididos em Gram-positivos ou Gram-negativos. Diante disto, torna-se importante a realização de um estudo para avaliação dos resultados desta terapia in vivo, para então questionar a necessidade de mudança em nossa primeira linha de tratamento, com intuito de melhorar os resultados e diminuir a morbidade visual.

\section{REFERÊNCIAS}

1. Schaefer F, Bruttin O, Zografos L, Guex-Crosier Y. Bacterial keratitis: a prospective clinical and microbiological study. Br J Ophthalmol. 2001;85(7):842-7.

2. Green M, Apel A, Stapleton F. A longitudinal study of trends in keratitis in Australia. Cornea. 2008:27(1):33-9.

3. Bennett HG, Hay J, Kirkness CM, Seal DV, Devonshire P. Antimicrobial management of presumed microbial keratitis: guidelines for treatment of central and peripheral ulcers. Br J Ophthalmol. 1998:82(2):137-45.

4. Butler TK, Spencer NA, Chan CC, Singh Gilhotra J, McClellan K. Infective keratitis in older patients: a 4 year review, 1998-2002. Br J Ophthalmol. 2005:89(5):591-6.

5. Bourcier T, Thomas F, Borderie V, Chaumeil C, Laroche L. Bacterial keratitis: predisposing factors, clinical and microbiological review of 300 cases. Br J Ophthalmol. 2003;87(7):834-8. Comment in: Br J Ophthalmol. 2003;87(7):805-6.

6. Kerautret J, Raobela L, Colin J. [Serious bacterial keratitis: a retrospective clinical and microbiological study]. J Fr Ophtalmol. 2006;29(8):883-8. French.

7. van der Meulen IJ, van Rooij J, Nieuwendaal CP, Van Cleijnenbreugel H, Geerards AJ, Remeijer L. Age-related risk factors, culture outcomes, and prognosis in patients admitted with infectious keratitis to two Dutch tertiary referral centers. Cornea. 2008;27(5):539-44.

8. Wang AG, Wu CC, Liu JH. Bacterial corneal ulcer: a multivariate study. Ophthalmologica. 1998;212(2):126-32

9. Tan DT, Lee CP, Lim AS. Corneal ulcers in two institutions in Singapore: analysis of causative factors, organisms and antibiotic resistance. Ann Acad Med Singapore. 1995; 24(6):823-9.

10. Sacramento RS, Castro L, Freitas D, Branco BC, Lima ALH, Vieira L, et al. Estudo dos fatores epidemiológicos e influentes na ceratite microbiana em serviço universitário. Rev Bras Oftalmol. 2005;64(1):7-13.

11. Green M, Apel A, Stapleton F. Risk factors and causative organisms in microbial keratitis. Cornea. 2008;27(1):22-7.

12. Levey SB, Katz HR, Abrams DA, Hirschbein MJ, Marsh MJ. The role of cultures in the management of ulcerative keratitis. Cornea. 1997;16(4):383-6. 
13. McLeod SD, Kolahdouz-Isfahani A, Rostamian K, Flowers CW, Lee PP, McDonnell PJ. The role of smears, cultures, and antibiotic sensitivity testing in the management of suspected infectious keratitis. Ophthalmology. 1996;103(1):23-8. Comment in: Ophthalmology. 1996; 103(8):1165-6

14. Rodman RC, Spisak S, Sugar A, Meyer RF, Soong HK, Musch DC. The utility of culturing corneal ulcers in a tertiary referral center versus a general ophthalmology clinic. Ophthalmology. 1997;104(11):1897-901.

15. Yeh DL, Stinnett SS, Afshari NA. Analysis of bacterial cultures in infectious keratitis, 1997 to 2004. Am J Ophthalmol. 2006;142(6):1066-8.

16. Rajpal $K$, Hall R, Long H, Wells A. Five-year experience of corneal scrapes at Wellington Eye Department, New Zealand. N Z Med J. 2007;120(1260):U2682.

17. Pachigolla G, Blomquist P, Cavanagh HD. Microbial keratitis pathogens and antibiotic susceptibilities: a 5-year review of cases at an urban county hospital in north Texas. Eye Contact Lens. 2007;33(1):45-9.

18. Dantas PE, Uesugui E, Nishiwaki-Dantas MC, Mimica $\sqcup$. Antibacterial activity of anesthetic solutions and preservatives: an in vitro comparative study. Cornea. 2000;19(3):353-4.

19. Uesugui E, Cypel-Gomes MC, Atique D, Goulart DG, Galucci FR, Nishiwaki-Dantas MC, Dantas PEC. Identificação laboratorial dos patógenos oculares mais frequentes e sua suscetibilidade in vitro aos agentes antimicrobianos. Arq Bras Oftalmol. 2002;65(3): 339-42.

20. Wakisaka E, Ferreira MA, Rocha FJ, Freitas LL, Guidugli T, Lima ALH. Cultura de material provindo de úlceras de córnea em laboratório de referência. Arq Bras Oftalmol. 1990;53(5): 196-200.
21. Fong CF, Hu FR, Tseng CH, Wang IJ, Chen WL, Hou YC. Antibiotic susceptibility of bacterial isolates from bacterial keratitis cases in a university hospital in Taiwan. Am J Ophthalmol. 2007;144(5):682-9.

22. Sirikul T, Prabriputaloong T, Smathivat A, Chuck RS, Vongthongsri A. Predisposing factors and etiologic diagnosis of ulcerative keratitis. Cornea. 2008;27(3):283-7.

23. Chaudhuri PR, Godfrey B. Treatment of bacterial corneal ulcers with concentrated antibiotic eye drops. Trans Ophthalmol Soc U K. 1982;102(Pt 1):11-4.

24. Hyndiuk RA, Eiferman RA, Caldwell DR, Rosenwasser GO, Santos Cl, Katz HR, et al. Comparison of ciprofloxacin ophthalmic solution $0.3 \%$ to fortified tobramycin-cefazolin in treating bacterial corneal ulcers. Ciprofloxacin Bacterial Keratitis Study Group. Ophthalmology. 1996;103(11):1854-62; discussion 1862-3.

25. O'Brien TP, Maguire MG, Fink NE, Alfonso E, McDonnell P. Efficacy of ofloxacin vs cefazolin and tobramycin in the therapy for bacterial keratitis. Report from the Bacterial Keratitis Study Research Group. Arch Ophthalmol. 1995;113(10):1257-65.

26. Kunimoto DY, Sharma S, Garg P, Rao GN. In vitro susceptibility of bacterial keratitis pathogens to ciprofloxacin. Emerging resistance. Ophthalmology. 1999;106(1):80-5.

27. Goldstein MH, Kowalski RP, Gordon YJ. Emerging fluoroquinolone resistance in bacterial keratitis: a 5-year review. Ophthalmology. 1999;106(7):1313-8.

28. Alexandrakis G, Alfonso EC, Miller D. Shifting trends in bacterial keratitis in south Florida and emerging resistance to fluoroquinolones. Ophthalmology. 2000; 107(8):1497-502.

29. Song A, McCulleyTJ, Lam BL, FeuerWJ, Miller D, Alfonso EC. Pseudomonas aeruginosa in vitro corneal isolate sensitivity to ofloxacin, ciprofloxacin, and trovafloxacin: a comparative study. Am J Ophthalmol. 2001;131(6):795-6. 